\title{
A Comparative Analysis of Use of Google Glass versus. GoPro Intraoperative Video Recording of Scleral Buckle Surgery
}

\author{
Rebecca R. Soares, MD ${ }^{1}$ Melissa R. Sieber, MD ${ }^{1} \quad$ Katherine E. Talcott, MD ${ }^{2} \quad$ Allen Chiang, MD ${ }^{1}$ \\ Sunir J. Garg, MD ${ }^{1}$ \\ ${ }^{1}$ The Retina Service of Wills Eye Hospital, MidAtlantic Retina, \\ Philadelphia, Pennsylvania \\ ${ }^{2}$ Cole Eye Institute, Cleveland Clinic, Cleveland, Ohio \\ J Acad Ophthalmol 2021;13:e124-e128.

\begin{abstract}
Address for correspondence Sunir J. Garg, MD, The Retina Service of Wills Eye Hospital, MidAtlantic Retina, Wills Eye Hospital, 840 Walnut Street, Suite 1020, Philadelphia, PA 19107 (e-mail: sgarg@midatlanticretina.com).
\end{abstract}

\begin{abstract}
Importance The scleral buckle technique is difficult to teach, given a decrease in the frequency of its use and the challenges of its video recording.

Background This study compares two different modalities for recording intraoperative videos of scleral buckle surgery.

Design Present study is a cross-sectional survey conducted in an academic hospital. Participants A total of 36 medical students, ophthalmologic residents, and vitreoretinal surgery fellows participated in this survey.

Methods Five scleral buckle surgeries were filmed simultaneously using Google Glass and GoPro. Single-masked survey of trainees comparing video and audio quality of 38 10 -second clips from each device, and listing the steps of scleral buckle surgery. Institutional review board approval and informed consent were obtained.

Main Outcome Measures Trainees were asked for grading the video device with respect to audio and video quality as favorable or unfavorable.

Results GoPro clips had more favorable ratings of video (73.3\%) and audio (75.9\%), compared with Google Glass (36.7 and 58.5\%, respectively; $p<0.005$ ). A total of $2.8 \%$

\section{Keywords}

- scleral buckle

- surgical teaching

- surgical video

- wearable device respondents listed the correct order of surgical steps in pretest, compared with $55.6 \%$ in posttest $(p<0.005)$.

Conclusion and Relevance Wearable recording devices can allow for video recording of scleral buckle surgery which has high utility in teaching trainees. GoPro and Google Glass each have distinct advantages.
\end{abstract}

For many years, scleral buckle surgery represented the goldstandard technique for management of rhegmatogenous retinal detachment. However, innovations in vitreoretinal surgery over the last few decades have shifted the surgical paradigm away from primary scleral buckling toward vitrec- tomy and vitrectomy combined with a scleral buckle. ${ }^{1} \mathrm{Nev}-$ ertheless, scleral buckle surgery remains an important surgical option, particularly for cases such as young, phakic patients, as an adjunct to vitrectomy in complex detachments, and in retinal dialysis-associated detachments. ${ }^{2}$ received

July 1,2020

accepted after revision

April 3, 2021
DOI https://doi.org/ $10.1055 / \mathrm{s}-0041-1733931$ ISSN 2475-4757.

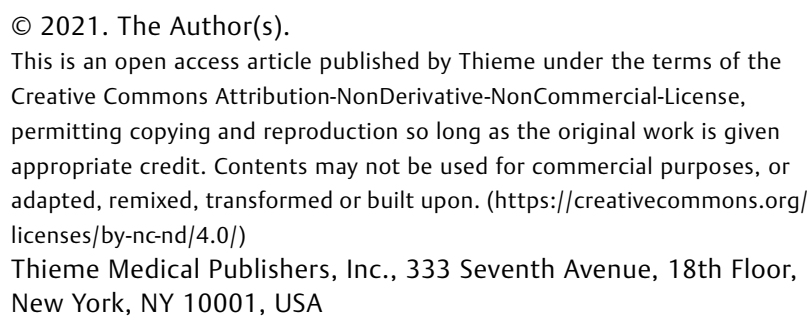


Teaching scleral buckling techniques to trainees has become more challenging due to the decreasing frequency of scleral buckle surgery, as well as by the challenges to sufficiently record and review the surgeries to optimize technique. During scleral buckle surgery, the use of an operating microscope with built-in digital recording capability can be unwieldy; surgical maneuvers during scleral buckle surgery often require the surgeon and the assistant to be mobile and work at an angle oblique to the central axis of a microscope. ${ }^{3}$ The traditional method of teaching scleral buckle surgery often requires the surgeon and trainees to crowd around the surgical eye, limiting the visibility and audibility of surgical instruction. Furthermore, this traditional method precludes video-assisted review and feedback.

First-person wearable video devices may provide a convenient way to record and teach scleral buckle surgery. Google Glass Explorer Edition (Google Inc, Mountain View, CA) was released in 2013 as an accessory worn similarly to a pair of glasses, but with a single small, interactive screen and camera positioned just above the user's visual axis. Although the device faced questions of usefulness for the general consumer and is no long sold commercially, it remains an important tool in the health care industry, especially in surgical education. ${ }^{4}$ GoPro HERO 4 (GoPro, Inc., San Mateo, CA) offers another option for surgical video recording. The mountable camera, introduced commercially as a camera for outdoor and athletic purposes, can be worn in the operating room to record surgery with the use of a head strap. ${ }^{5,6}$ Both cameras capably capture digital audio and video. As of yet, there have been few head-to-head comparisons of these wearable devices for recording and teaching surgery in any field of medicine or for recording ophthalmic surgery. $3,7-9$ There have been no large validation studies or comparative analyses of Google Glass and GoPro for use in ophthalmic surgery. The purpose this study was to (1) compare the audio and visual quality of intraoperative video recordings made with Google Glass and GoPro during scleral buckle surgery, and (2) determine the utility of intraoperative videos recorded with Google Glass versus GoPro for teaching scleral buckle surgery to ophthalmology trainees.

\section{Methods}

Institutional review board approval for this prospective, single-center cross-sectional study was obtained prior to conducting the study. The study was conducted in compliance with the Health Insurance Portability and Accountability Act, adhering to the principles of the Declaration of Helsinki.

Nine eyes undergoing primary rhegmatogenous retinal detachment repair with scleral buckle alone performed between 2015 and 2018 were recorded on two different hands-free video devices, GoPro and Google Glass (device specifications in - Table $\mathbf{1}$ ). The devices were worn simultaneously by a single attending operating surgeon, either A.C. or S.J.G., as they performed the surgery. Both video and audio were recorded simultaneously. The cases contained no identifying patient information. The videos included patients age 18 years or older undergoing scleral buckle surgery at Wills Eye Hospital for rhegmatogenous retinal detachment.

The five cases with the most complete videos from each device were chosen for inclusion in the survey to abbreviate the survey. Each case had at least seven of the nine salient steps filmed with both devices simultaneously of sufficient quality to be used for grading. One case had all nine salient steps. Four of the cases did not have comparable simultaneous videos for comparison, thus were excluded from further analysis. The cases were then trimmed into 76 10-second clips ( 38 Google Glass and 38 GoPro), each clip highlighting one of nine salient steps of scleral buckle

Table 1 Google Glass Explorer (Google Inc., Mountain View, CA) versus GoPro Hero 4 Black (GoPro, Inc., San Mateo, CA) device specifications

\begin{tabular}{|l|l|l|}
\hline Device specifications & Google Glass Explorer & GoPro Hero 4 Black \\
\hline Maximum video resolution & $720 \mathrm{p}$ & $1,080 \mathrm{p}$ \\
\hline High-definition video support & No & Yes, 4K \\
\hline Field of view & Wide, fixed & Ultrawide, medium, and narrow \\
\hline Video format & MP4 (H.264) & MP4 (H.264) \\
\hline White balance & None & Automatic and manual adjustments \\
\hline Low lux features & None & Low lux, night mode \\
\hline Microphone & Mono & Mono, wind-noise reduction features \\
\hline Connectivity & Wi-Fi and Bluetooth & Wi-Fi and Bluetooth \\
\hline Storage & $12 \mathrm{~GB}$ & MicroSDc \\
\hline Battery life & $\begin{array}{l}\text { Approximately 50-60 minutes of continuous } \\
\text { video recording }\end{array}$ & $\begin{array}{l}\text { Approximately 60-90 minutes of continuous } \\
\text { video recording (4K resolution) }\end{array}$ \\
\hline Weight & $42 \mathrm{~g}$ & $87 \mathrm{~g}$ \\
\hline Other features & $\begin{array}{l}\text { Prescription lenses may } \\
\text { be used in glasses }\end{array}$ & \\
\hline
\end{tabular}

Abbreviations: GB, gigabytes; p, pixels; SD, storage drive. 
Soares et al.

surgery: conjunctival peritomy, muscle isolation, suture pass, suture tie, buckle placement, Watzke's sleeve, and scleral suture. Professional editing software was used to crop, zoom, and center the operative eye, ensuring that clips from each device were edited the same way. No other postrecording adjustments or enhancements of the videos were made.

\section{Survey}

From August to October 2019, an online survey was sent to ophthalmology residents, retina fellows with the graduating year of 2018 to 2020, and current Thomas Jefferson University medical students involved in the Ophthalmology Interest Group. All respondents were 18 years of age and older. The study investigators were excluded. Respondents were recruited voluntarily and informed consent was obtained. The surveys involved a masked, side-by-side comparison of Google Glass and GoPro clips of the same surgical step, and asked the respondent to rate video and audio quality as "excellent," "good," “fair," or "poor" based on visualization of the anatomical area and surgical process and audio clarity; Google Glass clips were shown first and GoPro second. Additional questions asked to respondents to evaluate the utility of these surgical videos for education and videoassisted feedback. Respondents also completed a short multiple-choice quiz to identify the correct order of steps of scleral buckle surgery before and after viewing the videos. The full list of survey questions appears in -Supplementary Appendix S1 (available in the online version).

\section{Statistical Analysis}

Statistical analyses were conducted using SPSS 26 (Armonk, NY: IBM Corp.). Pre- and posttest scores and subjective level of respondent confidence were analyzed with McNemar's test and Wilcoxon's signed-rank test. Proportional data were tested for significance using Chi-square analysis. Repeated measures of continuous and categorical variables were compared using the generalized estimating equation with a binary logistic model of favorable ("good" and "excellent") and unfavorable ("poor" and "fair") responses. A $p$-value of $\leq 0.05$ was determined to be statistically significant.

\section{Results}

Baseline characteristics of 36 respondents are shown in -Table 2. Of respondents, $41.6 \%$ were ophthalmology residents, $47.2 \%$ were medical students, $2.8 \%$ were firstyear retina fellows, and $8.3 \%$ were second-year retina fellows.

For the 76 different surgical clips, from the 36 trainees, there were 5,349 ratings of audio and video quality. Regarding Google Glass, 1,273 of 2,676 (47.6\%) ratings were favorable ("good" or "excellent"), whereas 1,994 of 2,673 (74.6\%) ratings were favorable ( $p<0.005$, Chi-square) for GoPro.

Overall, 1,472 of 2,677 (55.0\%) ratings on video quality from either modality were favorable. Of ratings in regard to video quality, 981 of 1,338 (73.3\%) found GoPro clips to be favorable, compared with 491 of 1339 (36.7\%) ratings on Google Glass clips $(p<0.005)$.
Table 2 Baseline characteristics of survey respondents $(n=36)$

\begin{tabular}{|c|c|}
\hline \multicolumn{2}{|l|}{ Demographics } \\
\hline $\begin{array}{l}\text { Age }(y) \\
\text { Mean } \pm \text { SD }\end{array}$ & $27.5( \pm 2.7)$ \\
\hline \multicolumn{2}{|l|}{ Gender $(n=35)$} \\
\hline Male & $17(50 \%)$ \\
\hline \multicolumn{2}{|l|}{ Level of training of respondent } \\
\hline Medical student & $17(47.2 \%)$ \\
\hline PGY-1 & $0(0 \%)$ \\
\hline PGY-2 (first-year ophthalmology resident) & $4(11.1 \%)$ \\
\hline $\begin{array}{l}\text { PGY-3 (second-year ophthalmology } \\
\text { resident) }\end{array}$ & $4(11.1 \%)$ \\
\hline PGY-4 (third-year ophthalmology resident) & $4(19.4 \%)$ \\
\hline PGY-5 (first-year retina fellow) & $1(2.8 \%)$ \\
\hline PGY-6 (second-year retina fellow) & $3(8.3 \%)$ \\
\hline
\end{tabular}

Abbreviations: PGY, postgraduate year, year after graduation from medical school; SD, standard deviation.

Of ratings of audio quality from either modality, 1,795 of $2,672(67.2 \%)$ found audio clarity to be favorable. GoPro audio also had a more favorable response, with 1,013of 1,335 (75.9\%) ratings of good or excellent, compared with 782 of 1,337 (58.5\%) ratings about Google Glass audio $(p<0.005)$.

After adjusting for age, gender, training level, and surgeon and accounting for repeated measures with a generalized estimating equation binary regression model, type of video recorder still had a significant influence on favorable rating with regard to both video $(p<0.005)$ and audio quality $(p=0.003)$. Age, gender, and level of training of the respondent had no significant influence on the model for a favorable (good or excellent) rating. After adjustment for other abovementioned factors, attending surgeon recording the video did have a significant influence on favorable ratings for audio quality ( $p=0.002)$ and for video quality $(p=0.039)$.

Prior to taking the survey, respondents were asked to list the nine steps of scleral buckle surgery in the correct order given a dropdown menu. Only $1(2.8 \%)$ of 34 respondents who completed this pretest, listed all the steps in the correct order. Respondents were asked to rate their confidence in knowing the steps of scleral buckle surgery on a Likert's scale of 1 to 5 , with 1 being "not at all confident" to 5 being "very confident." Prior to the survey, participants had a mean confidence of $1.75 \pm 1.18$. After completing one full case comparing the Google Glass and GoPro clips of all nine scleral buckle steps, respondents were given a posttest asking for the correct order of scleral buckle steps. Of 36 who completed the posttest, 20 (55.6\%) listed all the steps in the correct order, a significantly higher proportion than the pretest ( $p<0.005$, McNemar's test). At the posttest, respondents reported a significant increase in mean confidence in scleral buckle steps to $3.36 \pm 1.13(p<0.005$, Wilcoxon's signed-rank test).

If made available, 32 of 36 (88.9\%) respondents would "always" or "very often" watch brief videos of operations 
being performed by attending surgeons before going into the operating room to perform the same operation. Furthermore, 31 of 36 (86.1\%) respondents found watching operative videos performed by others "extremely useful" or "very useful" in assisting in learning.

\section{Discussion}

Our study compared the video and audio quality obtained by Google Glass versus GoPro during scleral buckle surgery to explore their use for video-assisted teaching. Our study determined that GoPro had significantly more favorable responses than Google Glass for both video and audio quality. These differences persisted even when adjusting for trainee age, gender, level of training, and attending surgeon. This finding is in concordance with results of other head-to-head comparisons of device quality. ${ }^{7,8}$

Differences in the technical specifications of each device likely contributed to video and audio quality. GoPro Hero 4 videos were recorded in the 1,080 pixel high-resolution setting. Google Glass was filmed in its maximum resolution setting, 720 pixel. GoPro also has an automatic white-balance feature that improved washout in each of the videos while white balancing is not adjustable with Google Glass which has been previously reported as one of its consistent limitations in surgical settings. ${ }^{3}$ Furthermore, the GoPro has a variety of field-of-view options of which the "narrow view" was used for surgical filming. Google Glass, by comparison, has a wideangle view, leading to a smaller view of the operative field. ${ }^{3}$

The attending surgeon who performed the surgery and recorded the video and audio was significantly associated with video and audio rating. This highlights the fact that the quality of recording with each device is quite user dependent. GoPro Hero 4 can be worn midforehead. Google Glass mounts like a pair of eyeglasses. Users must adjust to pointing each device at the surgical field and bear the ergonomic weight of the hardware. Quality is likely associated with user-learning and ergonomic adaptability. One limitation of each device is inherent to first person recording, the camera only records what it is pointed at. Often during surgery, the surgeon moves their eyes more than the head to see the operative field, so if the surgeon did not maintain awareness about the direction of the camera relative to the operative field, the surgery may not have been effectively recorded. Of note, GoPro can connect to a smart-phone application which can be monitored by a third-party to see when the recording is off-center.

There are other notable differences between the two wearable devices. Google Glass has a shorter battery life. ${ }^{3,10}$ Partially to conserve battery life, Google Glass defaults to 10 -second long videos. To film a longer video, this default must be changed before each surgery. ${ }^{3}$ Since the initiation and completion of this study, both devices have had hardware updates that could further improve on the creation of surgical videos. GoPro Hero 8, the newest version of the device, has new attachable "mods," including a display screen that could be used to ensure centration during surgical filming. ${ }^{11}$ Google Glass announced its new "Enterprise
Edition 2" in 2019, which can be customized for professional use, has higher resolution and a slightly longer battery life. ${ }^{12}$

The pre- and posttest in this survey objectively demonstrated that not only did confidence in the steps of the surgery significantly increase, but also the percentage of respondents who could correctly order the steps significantly increased. This benefit of video review from wearable devices is consistent with other literature citing the importance of video review in surgical education. ${ }^{13,14}$ The majority of respondents felt watching self-created or other-created surgical videos was instrumental in their training, with a majority responding they would frequently watch videos created by attending surgeons prior to the same operation, and that they found watching surgical videos useful in their learning.

\section{Limitations}

Our study had several limitations. The fact that our videos were recorded simultaneously on both devices by the same surgeon did allow a greater standardization between the Google Glass and GoPro videos. However, it would be uncommon to wear both devices in a real-life setting and doing so may have compromised the video quality of both devices, as it was more difficult to keep both centered at the same time. The GoPro was more difficult to keep centered, as it is head mounted, so its favorability in terms of video quality may be disproportionately underestimated. Our nonrandom survey, which was voluntary and not part of standard educational evaluation, could have been affected by response bias. It is possible that those who did not respond were less likely to find surgical videos helpful. This could bias our results toward higher overall favorability of surgical videos. Google Glass videos were consistently shown first, which could potentially cause a bias leading to the respondent to classify the GoPro videos as greater or lesser quality because of the order in which they were viewed. Finally, our survey had a high percentage of medical student respondents, who may have had different standards for quality of visualization, given less exposure to ophthalmic surgery, specifically scleral buckle surgery.

\section{Conclusion}

Modern first-person wearable recording devices such as Google Glass and GoPro may make recording quality videos not only more feasible but also more convenient. Each recording device has its distinct advantages: GoPro appears to offer better video and audio quality and battery life, while Google Glass may be the more ergonomic option for prolonged use due to its lighter weight and ease of wear as glasses. The use of Google Glass or GoPro has previously proven valuable for strabismus and oculoplastic surgery. ${ }^{9}$ Our findings suggest that devices such as Google Glass and GoPro can facilitate surgical recording and improve education in scleral buckle surgery. Additional studies would be helpful to examine the usefulness of these types of recordings during surgeries for which an operating microscope is unavailable or unwieldy. 


\section{Financial Support}

This study was supported by the J. Arch McNamara Fund for Retina Research, MidAtlantic Retina, Philadelphia, PA.

\section{Conflict of Interest}

No conflicting relationship exists for any author. No proprietary interests in the material presented.

\section{References}

1 Ambati J, Arroyo JG. Postoperative complications of scleral buckling surgery. Int Ophthalmol Clin 2000;40(01):175-185

2 Thanos A, Papakostas TD, Young LH. Scleral buckle: does it still have a role in retinal detachment repair? Int Ophthalmol Clin 2015;55(04):147-156

3 Rahimy E, Garg SJ. Google Glass for recording scleral buckling surgery. JAMA Ophthalmol 2015;133(06):710-711

4 Wei NJ, Dougherty B, Myers A, Badawy SM. Using Google Glass in surgical settings: systematic review. JMIR Mhealth Uhealth 2018; 6(03): e54

5 Birnbaum FA, Wang A, Brady CJ. Stereoscopic surgical recording using GoPro cameras: a low-cost means for capturing external eye surgery. JAMA Ophthalmol 2015;133(12):1483-1484
6 Calvo CM, Sridhar J, Hong BK, Rahimy E, Hsu J, Garg SJ. Video recording of vitreous tap and intravitreal antibiotic injection from the surgeon's perspective. Retina 2015;35(10):2147-2149

7 Paro JAM, Nazareli R, Gurjala A, Berger A, Lee GK. Video-based self-review: comparing Google Glass and GoPro technologies. Ann Plast Surg 2015;74(Suppl 1):S71-S74

8 Lee CK, Kim Y, Lee N, Kim B, Kim D, Yi S. Feasibility study of utilization of action camera, GoPro Hero 4, Google Glass, and Panasonic HX-A100 in spine surgery. Spine 2017;42(04):275-280

9 Lin LK. Surgical video recording with a modified GoPro Hero 4 camera. Clin Ophthalmol 2016;10:117-119. Doi: 10.2147/OPTH.S95666

10 Ho VY, Shah VG, Yates DM, Shah GK. GoPro HERO 4 Black recording of scleral buckle placement during retinal detachment repair. Can J Ophthalmol 2017;52(04):416-418

11 HERO8 Black Accessed November 18, 2019 at: https://gopro. com/en/us/shop/cameras/hero8-black/CHDHX-801-master.html

12 Glass. Accessed November 18, 2019 at: https://www.google.com/ glass/start/

13 Farquharson AL, Cresswell AC, Beard JD, Chan P. Randomized trial of the effect of video feedback on the acquisition of surgical skills. Br J Surg 2013;100(11):1448-1453

14 Hamad GG, Brown MT, Clavijo-Alvarez JA. Postoperative video debriefing reduces technical errors in laparoscopic surgery. Am J Surg 2007;194(01):110-114 